\title{
Optimal Parameter Selection for Image Watermarking Using MOGA
}

\author{
Dinabandhu Bhandari, Lopamudra Kundu, and Sankar K. Pal \\ Center for Soft Computing Research, Indian Statistical Institute, Kolkata, India \\ dinabandhu.bhandari@gmail.com
}

\begin{abstract}
The notion of the proposed methodology is to optimize multidimensional nonlinear problem of conflicting nature that exists among imperceptibility and robustness in image watermarking. The methodology exploits the potentiality of Multi-Objective Genetic Algorithm (MOGA) in searching multiple non-dominated solutions lying on the Pareto front. The characteristics curve of the image are then analyzed and the most appropriate solution is selected using a merit function defined over evaluation measures. The efficacy of the suggested method is demonstrated by reporting the resultant watermarked images and restored watermarks extracted from their mean and median filtered versions.
\end{abstract}

Keywords: Image Characteristics Curve, Merit Function, Imperceptibility, Robustness, SSIM.

\section{Introduction}

Digital Watermarking, which is a digital adaptation of the traditional visible paper watermark, is an important sub-discipline of information hiding. Though visible digital watermarks are in use, most of the research works are centered on imperceptible digital watermarking that has wider applications [8]. It includes copyright protection, traitor tracking using fingerprinting, copy protection in digital multimedia distribution systems, image authentication and many more. Commonly used digital watermarking techniques for images include LSB (least significant bit) replacement, the patchwork algorithm by Bender et al. [12], predictive coding, discrete Fourier transform, discrete cosine transform, MellinFourier transform [1], hierarchical watermark extraction process based on wavelet transformation by Xia et al.14, spread spectrum technique [10, phase modulation, amplitude modulation etc. Progressive watermarking technique in DCT (Discrete cosine transform) domain was proposed by Huang et al. where genetic algorithm was used to search the optimal frequency band for embedding of watermark while improving simultaneously security, robustness and visual quality of the watermarked image [7].

Two major tasks are involved in fulfilling these requirements:

1. Selection of an appropriate modulation/mapping function (reversible) to embed the auxiliary image into the cover image for obtaining a desired output. Usually, a suitable nonlinear reversible mapping function is used.

2. Selection of objective functions to evaluate the embedded image. 
Given the images (cover and auxiliary), it is difficult to select a modulation function which will be best suited without prior knowledge of the image statistics 3. Even if we are given the image statistics, it may be possible to estimate only approximately the function required for watermarking. Moreover, to make the evaluation process objective, it is necessary to define appropriate objective functions that will quantitatively measure the embedded image with respect to imperceptibility and robustness [2].

The effectiveness of multi-objective genetic algorithm (MOGA) is exploited to find a number of solutions in the Pareto-optimal front. Automatic selection of an optimum solution is made using the image characteristics curve (ICC) that follows the concept of operating characteristics curve (OCC). In this context, the merit of a solution is calculated taking into account the effect of evaluation measures and their importance [4]. The merit of a solution is derived keeping in mind the simultaneous optimization of multiple objectives and their characteristics.

\section{Proposed Methodology}

The proposed methodology consists of 3 major parts.

1. Selection of embedding region and modulation function.

2. Selection of evaluation measures and formulation of MOGA.

3. Automatic selection of suitable solution.

Embedding region and modulation function: The embedding region is determined based on the distribution of the gray values of the cover image. The region from the cover image can be selected from its histogram on the basis of higher frequency of occurrence in pixel values in either end or on the basis of the histogram of the difference matrix [9]. A mask, equal in size as that of the auxiliary image, is used to search dynamically for the proper window where the watermark can be embedded. Once the embedding region is chosen, proper modulation function is used to embed the watermark. Most commonly used modulation functions [9] are:

- Power-law function: $x_{m+i, n+j}^{\prime}=A\left(a_{i, j}+\epsilon\right)^{\mu}$.

- Parabolic function: $x_{m+i, n+j}^{\prime}=A\left(1+\mu \sqrt{\left(a_{i, j}+\epsilon\right)}\right)$.

Where $A, \epsilon$ and $\mu$ are parameters of modulation functions.

Estimation of parameters using MOGA and formulation of objective functions: To estimate the parameters of the modulation function, mentioned above, the non-dominated sorting algorithm (NSGAII) is adopted in implementing the proposed methodology [11. Real coded genetic algorithm is used, where a string of 3 real numbers is considered as chromosome. Imperceptibility, robustness against mean and median filtering are the three objective functions considered in the implementation of the algorithm. They are defined as follows: Imperceptibility (Imp):

$$
\frac{\sum_{i=1}^{M} \sum_{j=1}^{N}\left|x_{m+i, n+j}^{\prime}-x_{m+i, n+j}\right|}{M . N}
$$


Robustness against mean filtering (R(mean):

$$
\frac{\sum_{i=1}^{M} \sum_{j=1}^{N} \mid x_{m+i, n+j}^{\prime}(\text { mean })-a_{i, j} \mid}{M . N}
$$

Robustness against mean filtering (R(median):

$$
\frac{\sum_{i=1}^{M} \sum_{j=1}^{N} \mid x_{m+i, n+j}^{\prime}(\text { median })-a_{i, j} \mid}{M . N}
$$

Where, $(M \times N)$ is dimension of the auxiliary image, $x_{i, j}, a_{i, j}$ and $x_{i, j}^{\prime}$ respectively are gray values of $(i, j)$ th pixel of the cover, auxiliary and embedded images. $x_{i, j}^{\prime}$ (mean) and $x_{i, j}^{\prime}$ (median) are respectively the mean and median of the $(3 \times 3)$ neighborhood of the $(i, j)$ th pixel of the watermarked image. Watermark recovery process uses inverse transformation function that maps $x_{m+i, n+j}^{\prime}$ into $x_{i, j}$ and thus the embedded image can be retrieved.

Automatic selection of suitable solution: The characteristics with respect to the objective functions of the non-dominated solutions contained in the archive are analyzed in selecting an appropriate and pragmatic solution. It is clear that a non-dominated solution optimizing one function does not optimize all others. Therefore, it is wise to select intuitively a solution that produces lower (near optimal) values for all the objective functions taken into account. To select such a suitable solution, a merit function can be defined taking into account the effect of all the objective functions with appropriate importance. In order to make a comparison between the decrease/ increase between the values of different functions, a better indicator is to divide these decrease/ increase by the range of the functions. This intuition helps to define a merit function $(\mu)$ for $r$ objective functions as follows:

$$
\mu(s)=\sum_{i=1}^{r} \frac{f_{i}(s)-f_{i, \min }}{f_{i, \max }-f_{i, \min }},
$$

Where, $f_{i}(s)$ is the value of the $i$ th objective function for string $s, f_{i, \min }$ and $f_{i, \max }$ respectively are the minimum and maximum values of the $i$ th function among all strings present in the archive. The maximum and minimum values of the functions are taken with respect to the explored non-dominated strings belonging to the archive.

\section{Implementation and Results}

The proposed algorithm is implemented by embedding an auxiliary image into a cover image and extracting the watermark from mean and median filtered versions of watermarked image. Power law function is used as the modulation function. The domains of the parameters considered are $A \in[0,10], \epsilon \in[0,0.1]$ and $\mu \in[0,1]$. For both mean and median filtering, $(3 \times 3)$ window is used. The effectiveness of the methodology has been demonstrated here for two cover images :Bandon beach(Bandon) $($ size $(610 \times 403))$ and Fontaine des Terreaux(Terreaux) 
$($ size $(768 \times 512))$ and two auxiliary images (watermark): Intel logo (size $(32 \times 16))$ and IAPR logo(size $(44 \times 18))$, depicted in fig. 1] [5]. Imperceptibility and Robustness defined in (1)-(3) are taken as the objective functions. The genetic parameters used for MOGA based watermarking method are: Size of population and archive $=20$, Number of generations $=500$, Probability of cross-over $=0.8$ and Probability of mutation $=0.1$. The algorithm is executed several times $(500$ generations for each run)for a pair of cover and auxiliary image. In each run we get 20 different sets of parameter values.

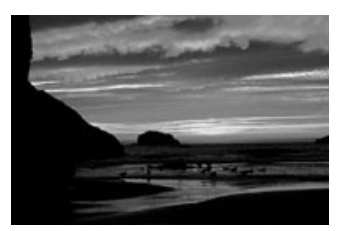

(a)

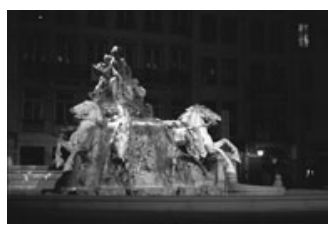

(b)

\section{inted IAPRe?}

(c) (d)

Fig. 1. Cover Images - (a) Bandon and (b) Terreaux; Watermark Images - (c) Intel and (d) IAPR

Fig. 2(a) depicts image characteristics curve for 20 different set of solutions for a particular run when Bandon is the cover image and Intel is the embedded logo. The graph shows how the objective function values vary with different solutions. A closer look at ICC will clearly reveal that all the solutions from 12 to 15 produce low values for all the three objective functions and may therefore appear comparable. Further investigation shows that merit function value corresponding to solution number 14 is minimum. The watermarked image obtained using the solution 14, and extracted watermarks after mean and median filtering of the embedded image are shown in fig $2(\mathrm{~b}), 2(\mathrm{~d})$. The resultant images show that the parameter values corresponding to minimum merit function value can produce high quality watermarked image in terms of imperceptibility and robustness. This proves indeed that merit function value can estimate properly the most suitable solution among the set of non-dominated solutions.

Similar results (not presented here for space scarcity) are obtained for other combinations of cover and watermark images mentioned before, viz., Bandon IAPR, Terreaux - Intel and Terreaux - IAPR. Visual inspection of these 3 sets of ICC curves reveal similar scenario as explained before. Same set of watermark images are embedded in several other cover images available in [5] and found similar performance of the proposed methodology.

To validate the results obtained, we have calculated SSIM between a given pair of cover and watermarked images for different solutions to investigate the variation of SSIM index with merit function value. Greater is the SSIM index, better will be the imperceptibility of the watermarked image [136]. The SSIM measures for imperceptibility and robustness separately and the combined SSIM index for four sets of images are given in Table 1. The combined SSIM indices 


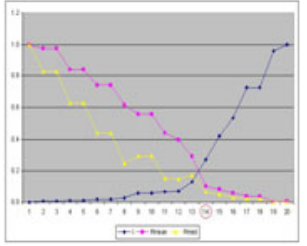

(a)

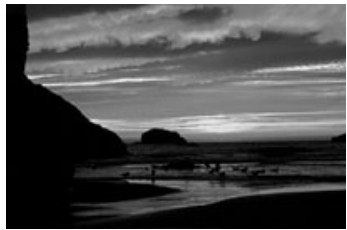

(b)

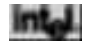

(c)

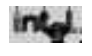

(d)

Fig. 2. Watermarked image and extracted watermarks for Bandon and Intel together with ICC

Table 1. SSIM measures for different sets of cover and watermark images

\begin{tabular}{|c|c|c|c|c|c|c|}
\hline Cover & Watermark & Soln. & SSIM(I) & SSIM(Rm) & SSIM(Rmd) & Combined \\
\hline Bandon & Intel & $\mathbf{1 4}$ & $\mathbf{0 . 9 9 9 7}$ & $\mathbf{0 . 6 1 4}$ & $\mathbf{0 . 7 5 7 6}$ & $\mathbf{2 . 3 7 1 3}$ \\
$"$ & $"$ & 7 & 0.9998 & 0.1995 & 0.5911 & 1.7904 \\
$"$ & $"$ & 11 & 0.9993 & 0.623 & 0.7351 & 2.358 \\
$"$ & $"$ & 13 & 0.9997 & 0.3508 & 0.5988 & 1.9493 \\
\hline Bandon & IAPR & $\mathbf{1 6}$ & $\mathbf{0 . 9 9 9 6}$ & $\mathbf{0 . 4 3 7 7}$ & $\mathbf{0 . 5 3 0 8}$ & $\mathbf{1 . 9 6 8 1}$ \\
$"$ & $"$ & 19 & 0.9995 & 0.0126 & 0.1909 & 1.203 \\
$"$ & $"$ & 15 & 0.9968 & 0.434 & 0.5336 & 1.9644 \\
$"$ & $"$ & 13 & 0.9993 & 0.0398 & 0.0246 & 1.0637 \\
\hline Terraux & Intel & $\mathbf{1 5}$ & $\mathbf{0 . 9 9 9 8}$ & $\mathbf{0 . 7 0 0 7}$ & $\mathbf{0 . 7 5 8 9}$ & $\mathbf{2 . 4 5 9 4}$ \\
$"$ & $"$ & 16 & 0.9998 & 0.6853 & 0.766 & 2.4511 \\
$"$ & $"$ & 14 & 0.9998 & 0.6956 & 0.7595 & 2.4549 \\
\hline Terraux & IAPR & $\mathbf{1 7}$ & $\mathbf{0 . 9 9 9 8}$ & $\mathbf{0 . 4 9 1 9}$ & $\mathbf{0 . 5 6 8 6}$ & $\mathbf{2 . 0 6 0 3}$ \\
$"$ & $"$ & 4 & 0.9998 & 0.497 & 0.5479 & 2.0447 \\
$"$ & $"$ & 16 & 0.9998 & 0.5006 & 0.5526 & 2.053 \\
$"$ & $"$ & 19 & 0.9998 & 0.5006 & 0.5526 & 2.053 \\
\hline
\end{tabular}

corresponding to different solutions reveal that the individual SSIM indices for imperceptibility, robustness against mean and median filtering may not be maximum for the preferred solution, but the combined SSIM index is maximum for the solution corresponding to minimum merit function value. This indeed proves quantitatively that merit is a good measure in choosing the best optimum solution among the set of non-dominated solution which optimizes both imperceptibility and robustness and can produce high quality watermarked images. The distortion is introduced with the help of Stirmark benchmark software that is a generic tool used for introducing attacks on an image like JPEG compression, Gaussian noise, non-linear filtering, cropping, rotation etc.

\section{Conclusions}

The effectiveness of MOGA in the automatic selection of modulation operator is tested for various cover and watermark images. The algorithm determines number of optimum parameter sets rather than a single one in selecting an appropriate modulation function. Afterwards, The image characteristics curve and merit function are used to select automatically the best one among the optimal parameter sets. Further work may be carried out to extend the proposed concept involving different transformation and objective functions. 


\section{Acknowledgements}

Authors sincerely acknowledge Prof. Malay K. Kundu of MIU, Indian Statistical Institute for constructive discussion and suggestion. Sankar K. Pal acknowledges the J. C. Bose National Fellowship of the Government of India.

\section{References}

1. Ruanaidh, J.J.K.Ó., Pun, T.: Rotation, translation and scale invariant digital image watermarking. In: Proceedings of the International Conference on Image Processing, Santa Barbara, California, October 1997, vol. 1, pp. 536-539 (1997)

2. Bhandari, D., Murthy, C.A., Pal, S.K.: Image enhancement using multi-objective genetic algorithms. In: Chaudhury, S., Mitra, S., Murthy, C.A., Sastry, P.S., Pal, S.K. (eds.) PReMI 2009. LNCS, vol. 5909, pp. 309-314. Springer, Heidelberg (2009)

3. Ekstrom, M.P.: Digital image processing techniques. Academic Press, New York (1984)

4. Hansen, M.P., Jaszkiewicz, A.: Evaluating the quality of approximations to the nondominated set. Technical Report Technical Report IMM-REP-1998-7, Institute of Mathematical Modelling, Technical University of Denmark (1998)

5. http://www.cl.cam.ac.uk/fapp2/watermarking

6. http://www.ece.uwaterloo.ca/z70wang/research/ssim/

7. Huang, H.C., Pan, J.S., Huamg, Y.H., Huang, K.C.: Progressive watermarking techniques using genetic algorithms. Circuits, Systems and Signal Processing 8, $58-68(2007)$

8. Katzenbeisser, S., Petitcolas, F.A.P.: Information hiding techniques for steganography and digital watermarking. Artech House Publishers (2000)

9. Maity, S.P., Kundu, M.K.: Genetic algorithms for optimality of data hiding in digital images. Soft Computing 13(4), 361-373 (2009)

10. Schilling, D.L., Pickholtz, R.L., Millstein, L.B.: Theory of spread spectrum communications-a tutorial. IEEE Transactions on Communications 30(5), 855-884 (1982)

11. Srinivas, N., Deb, K.: Multiobjective function optimization using nondominated sorting genetic algorithms. Evolutionary Computation 2(3), 221-248 (1995)

12. Gruhl, D., Bender, W., Morimoto, N.: Techniques for data hiding. In: Proceedings of the SPIE 2420, Storage and Retrieval for Image and Video Databases III, pp. 164-173 (1995)

13. Wang, Z., Bovik, A.C., Sheikh, H.R., Simoncelli, E.P.: Image quality assessment: From error visibility to structural similarity. IEEE Transactions on image processing 13(4), 600-612 (2004)

14. Xia, X.G., Boncelet, C.G., Arce, G.R.: A robust digital image watermarking method using wavelet-based fusion. In: Wavelet Transform Based Watermark for Digital Images, vol. 3, pp. 497-511. Optics Express (1998) 